\title{
Current practice in thoracic neoplasm diagnosis, evaluation and treatment
}

Despite increasing public awareness of smoking related morbidity and mortality and implementation of lung cancer screening (LCS) programs, lung cancer remains the number one cause of cancer related death (1). As our knowledge of lung cancer pathogenesis advances, approaches to diagnose, surveil and treat lung cancer also evolve. In this focus issue, we seek to update our readers on contemporary practices in thoracic neoplasm diagnosis, evaluation and treatment.

LCS has been proven to aid early cancer detection and reduce disease specific mortality. The article "Implication of new lung RADS on lung cancer screening" discusses the changes and additions made to the new LCS reporting system (LungRADS 1.1) while highlighting several challenges and opportunities as Lung-RADS version 1.1 is implemented. Leveraging recent advances in both hardware and software, artificial intelligence (AI) has taken lung cancer diagnosis to the next level. "Putting artificial intelligence (AI) on the spot: machine learning evaluation of pulmonary nodules" highlights this advancement in pulmonary nodule detection and characterization, with the ultimate goal of lung cancer prediction, while outlining some of the pitfalls and challenges that remain before bringing such progress to routine clinical use. Challenging the past imaging paradigm of conventional anatomic imaging techniques, positron emission tomography (PET) images physiology and has become an indispensable tool in oncology. Its contemporary role will be discussed in "Thoracic positron emission tomography: ${ }^{18}$ F-fluorodeoxyglucose and beyond". Pathology is the definitive step in cancer diagnosis. Unlike traditional invasive biopsies, liquid biopsy requires minimal to no tissue violation and has taken on an increasingly important role. Cecchini et al. discuss the contemporary role of liquid biopsy in their article "Liquid biopsy is a valuable tool in the diagnosis and management of lung cancer".

Advancements in imaging and radiotherapy (RT) techniques have allowed for remarkably precise delivery of high radiation dose per treatment fraction to intrathoracic targets. However, due to proximity of vital structures, targeted treatment of central and ultra-central thoracic tumors remains challenging. Owen et al. examine the contemporary practice of hypofractionated RT or stereotactic body radiation therapy (SBRT) for central and ultra-central thoracic tumors in "SBRT for central and ultra-central node negative lung cancer". An example of an ultra-central tumor is esophageal neoplasm. In "Proton beam therapy for esophagus cancer", the authors discuss the rationale, dosimetric and current clinical studies comparing outcomes of photon- and proton-based techniques (PBT), and future directions of PBT as a means of reducing toxicity and improving oncologic outcomes in esophageal cancer patients. Instead of being a monotherapy, RT has been increasingly shown to act synergistically with immunotherapy (IO), improving patient outcomes. Breen et al. describe the evidence for the evolving role and synergistic mechanisms of combining IO with RT in "Radiation and immunotberapy: emerging mechanisms of synergy".

With the advent of IO, options for patients with advanced lung cancers have expanded and the contemporary management of this population will be discussed in "Changing paradigm in advanced and metastatic lung cancer". Immunotherapies are well tolerated in general. However, due to the potential for severe immune-related adverse events in patients with pre-existing autoimmune diseases (AID), this population has been mostly excluded from immunotherapies. "Immunotherapy in patients with autoimmune disease" reviews the current evidence for safety and activity of IO in patients with pre-existing AID. Besides chemo- and radiotherapies, thermal ablation is an option for inoperable tumors and Lin et al. discuss current features and application of these ablation techniques in lung cancer treatment in "The current role and practice of lung ablation".

It is the sincere hope of this issue's authors that their work will stimulate further research and foster adoption of new technologies that will advance the field of lung cancer, ultimately improving clinical outcomes.

\section{Acknowledgments}

Funding: None. 


\section{Footnote}

Provenance and Peer Review: This article was commissioned by the editorial office, fournal of Thoracic Disease. The article did not undergo external peer review.

Conflicts of Interest: The author has completed the ICMJE uniform disclosure form (available at http://dx.doi.org/10.21037/ jtd-2019-cptn-11). The series "Contemporary Practice in Thoracic Neoplasm Diagnosis, Evaluation and Treatment" was commissioned by the editorial office without any funding or sponsorship. CWK served as the unpaid Guest Editor of the series and serves as an unpaid editorial board member of fournal of Thoracic Disease from Dec 2018 to Nov 2020. The author has no other conflicts of interest to declare.

Ethical Statement: The author is accountable for all aspects of the work in ensuring that questions related to the accuracy or integrity of any part of the work are appropriately investigated and resolved.

Open Access Statement: This is an Open Access article distributed in accordance with the Creative Commons AttributionNonCommercial-NoDerivs 4.0 International License (CC BY-NC-ND 4.0), which permits the non-commercial replication and distribution of the article with the strict proviso that no changes or edits are made and the original work is properly cited (including links to both the formal publication through the relevant DOI and the license). See: https://creativecommons.org/licenses/by-nc$\mathrm{nd} / 4.0 /$.

\section{References}

1. American Cancer Society. Cancer Facts \& Figures 2019. Atlanta: American Cancer Society, 2019.

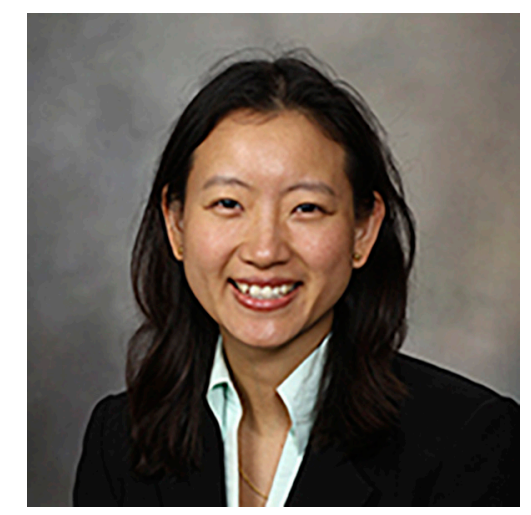

Chi Wan Koo

Chi Wan Koo, MD

Department of Radiology, Mayo Clinic, Rochester, MN, USA. (Email: koo.chiwan@mayo.edu) Submitted Jul 14, 2020. Accepted for publication Aug 17, 2020. doi: $10.21037 /$ jtd-2019-cptn-11

View this article at: http://dx.doi.org/10.21037/jtd-2019-cptn-11

Cite this article as: Koo CW. Current practice in thoracic neoplasm diagnosis, evaluation and treatment. J Thorac Dis 2020;12(11):6952-6953. doi: 10.21037/jtd-2019-cptn-11 